\title{
Physical simulation of dry microburst using impinging jet model with swirl
}

\author{
K. K. Das ${ }^{1 *}$, A. K. Ghosh ${ }^{2}$, K. P. Sinhamahapatra ${ }^{3}$ \\ ${ }^{1 *}$ Department of Mechanical Engineering, Assam Engineering College,Guwahati, INDIA \\ ${ }^{23}$ Department of Aerospace Engineering, Indian Institute of Technology Kharagpur, INDIA \\ "Corresponding Author: e-mail: kkdas1971@gmail.com, Tel+91-09864405087
}

\begin{abstract}
Severe thunderstorm is an important weather phenomena, which impact on aviation, space vehicle launching, agriculture in addition to its damage potential to life and properties. One of the most important event in the thunderstorms is the "Downburst". It consists of slow rotating column of air, which burst violently after reaching the ground. It is believed that a downburst is generated when the upward moving moist buoyant air can no longer be sustained above and subsided into downdraft. The flow due to downburst impacts on the ground and spreads outward in different directions. Downbursts are classified as either microburst or macroburst depending on their horizontal extent of damage. In this work, an attempt has been made to simulate the dry microburst (microburst not accompanied by rain) experimentally using the impinging jet model for investigating the macroflow dynamics and scale (Reynolds number) dependency of the downburst flow. Flow visualization is done using a smoke generator for understanding the flow dynamics.
\end{abstract}

Keywords: Microburst, Experimental simulation, Ring vortex, Macro-flow dynamics.

\section{Introduction}

The famous atmospheric scientist, Fujita (1981) has observed and studied the flow due to downburst impacting on the ground and spreading outward in the different directions. He classified downburst as either microburst or macroburst depending on their horizontal extent of damage. Physical modeling of downbursts has several advantages and disadvantages. One of physical modeling's major advantages is the fact that air itself is used for experimentation. This helps to minimize errors, caused due to incorrect modeling of the test fluid. Modeling with air as the test fluid has produced relatively good representation of the full scale phenomenon. However, it is very difficult to model the true downburst due to the complexity of the event. One of the first physical model of a thunderstorm downburst was made by Fujita at the University of Chicago in the 1970's (Fujita, 1990). Fujita's laboratory model consists of a buoyantly impinging plume impacting a surface, with the front edge made visible by dry-ice smoke. For the complexity of the full scale phenomenon, the physical simulation of the downburst is confined to the generic experiments of density currents impinging on a wall. Alahyari and Longmire (1995), Lundgren et al. (1992), Cooper et al. (1993), Didden and Ho (1985), Knowles and Myszko(1998) have studied experimental simulation of the downburst. Letchford and Chay(2002), Chay and Letchford(2002) and Sengupta and Sarkar (2007) performed physical modelling to study the flow field characteristics and pressure distribution the stationary and translational downburst. Numerical simulation of the downburst is performed by Proctor(1988),Craft et al. (1993) and Selvam and Homes(1992). Kim and Hangan(2006) and Sengupta and Sarkar (2007) simulated the downburst flow field with different turbulence model using FLUENT software. The primary objective of the present work is (a) to develop a physical simulator based on the impinging jet model to study the wind field characteristics of the microburst (b) to study the flow dynamics due to the variation of the $\mathrm{H} / \mathrm{D}_{\text {jet }}$ ratio(plate separation) and the jet velocity. (c) to study the flow patterns using flow visualization. 


\section{Physical modeling}

For the physical simulation of the stationary axisymmetric dry microbursts impinging jet model is considered and the experimental investigations are carried out in an impinging jet facility fabricated at the Wind Tunnel laboratory of the Aerospace Engineering Department, IIT Kharagpur with a $165 \mathrm{~mm}$ exit diameter pipe. Two $1.5 \mathrm{HP}$ centrifugal blowers are used to generate the impinging jet with swirl. Rotation is imparted to the jet using two cross jets having diameter $20 \mathrm{~mm}$ mounted in the main pipe as shown in figure 3(b) as full scale downburst have slow rotation due to the rotation of the parent storm. The velocity of the cross jets are kept fixed at $2 \mathrm{~m} / \mathrm{s}$ for the entire experimentation. Blower is connected to a bell mouth and the flow is directed through a pipe ensuring a fully developed turbulent flow. The dimension of the wooden platform on which the jet impinges is $1.5 \mathrm{~m} \times 1.5 \mathrm{~m}$ with roughness of 4.2 micron. The distance of the jet from the impinging platform $(H)$ can be varied between $125 \mathrm{~mm}$ and $400 \mathrm{~mm}$ using an adjustable frame to change the value of $\mathrm{H} / \mathrm{D}_{\text {jet }}$. The $\mathrm{H} / \mathrm{D}_{\text {jet }}$ ratios considered for this work are 1.0, 1.5,2.0. Henceforth, these ratios are referred as $R_{1}, R_{2}$ and $R_{3}$ respectively. Three jet velocities $\left(V_{\text {jet }}\right)$ of $10 \mathrm{~m} / \mathrm{s}, 15 \mathrm{~m} / \mathrm{s}$ and $20 \mathrm{~m} / \mathrm{s}$ are used in the experiments. These velocities are designated as $\mathrm{V}_{1}, \mathrm{~V}_{2}$ and $\mathrm{V}_{3}$ respectively. The three velocity magnitudes $V_{f e s}=\mathrm{V}_{1}, \mathrm{~V}_{2}$ and $\mathrm{V}_{3}$ correspond to Reynolds number of $1.1 \times 10^{5}, 1.6 \times 10^{5}$ and $2.2 \times 10^{5}$ respectively. Henceforth, these Reynolds numbers are represented by $\mathrm{Re}_{1}, \mathrm{Re}_{2}$ and $\mathrm{Re}_{3}$ respectively. Pressures at different points on the surface is measured by pressure transducer and multi tube manometer. Experimental readings are taken for various values of $\left(\mathrm{H} / \mathrm{D}_{\text {jet }}\right)$ and jet velocity $\left(\mathrm{V}_{\text {jet }}\right)$. Experimental setup is shown in figure 1.

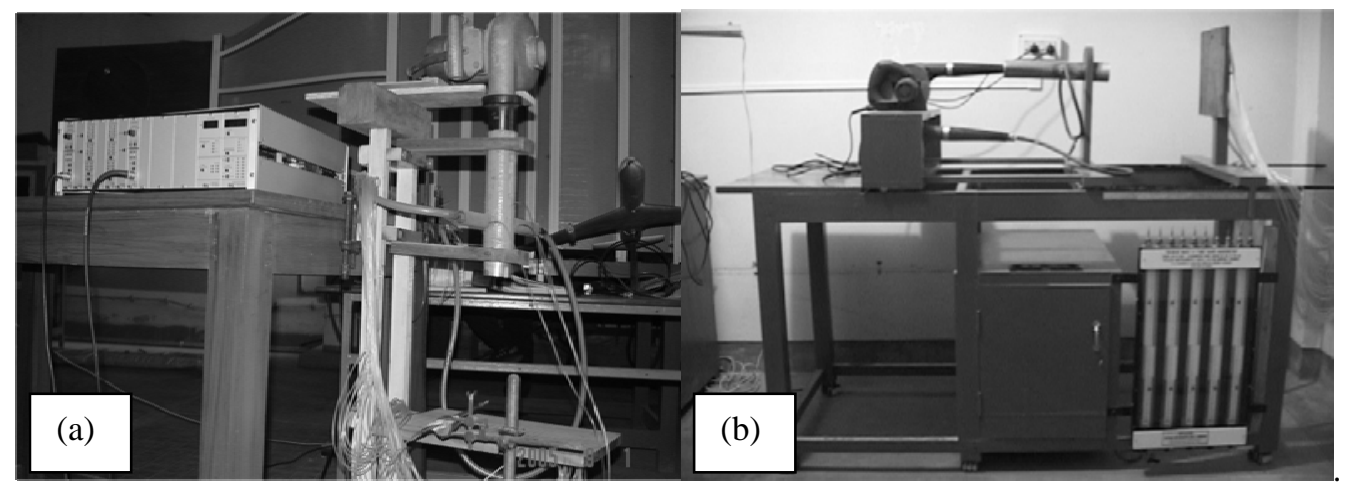

Figure 1 Physical simulator of the thunderstorm microburst fabricated at IIT Kharagpur

\subsection{Velocity and Pressure measurements}

A DANTEC 56C17 hot wire anemometer system is used to measure the velocity in the flow field. In addition, a vane type digital anemometer is also used to measure the velocity at some locations in the flow field. To determine the velocity profiles in the radial and axial directions, hot wire anemometer probe is placed in the DANTEC traversing system as shown in figure 2. Pressure is measured using a PDCR23 pressure transducer system with a scanivalve. To estimate the pressure on the impinging platform 300 pressure taps are placed on the platform. Pressure taps are connected to the scanivalve through $1 \mathrm{~mm}$ diameter PVC tubing. A multi tube manometer is also used to verify the pressure readings of the PDCR23 pressure transducer system.

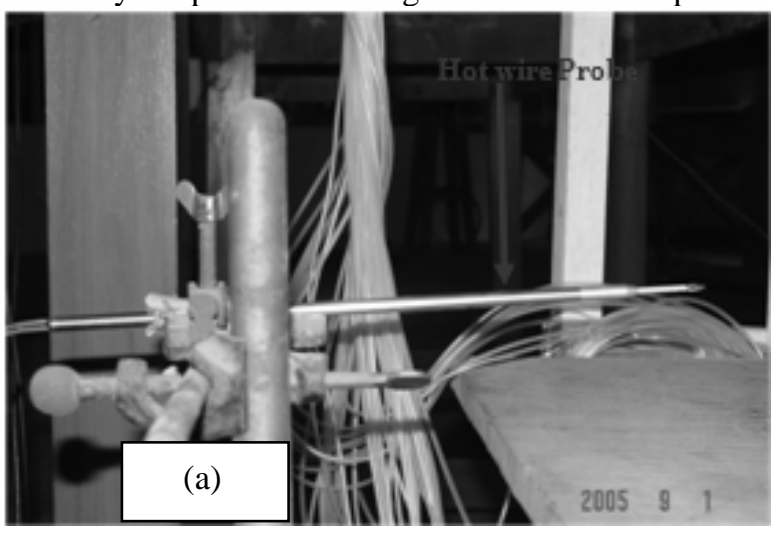



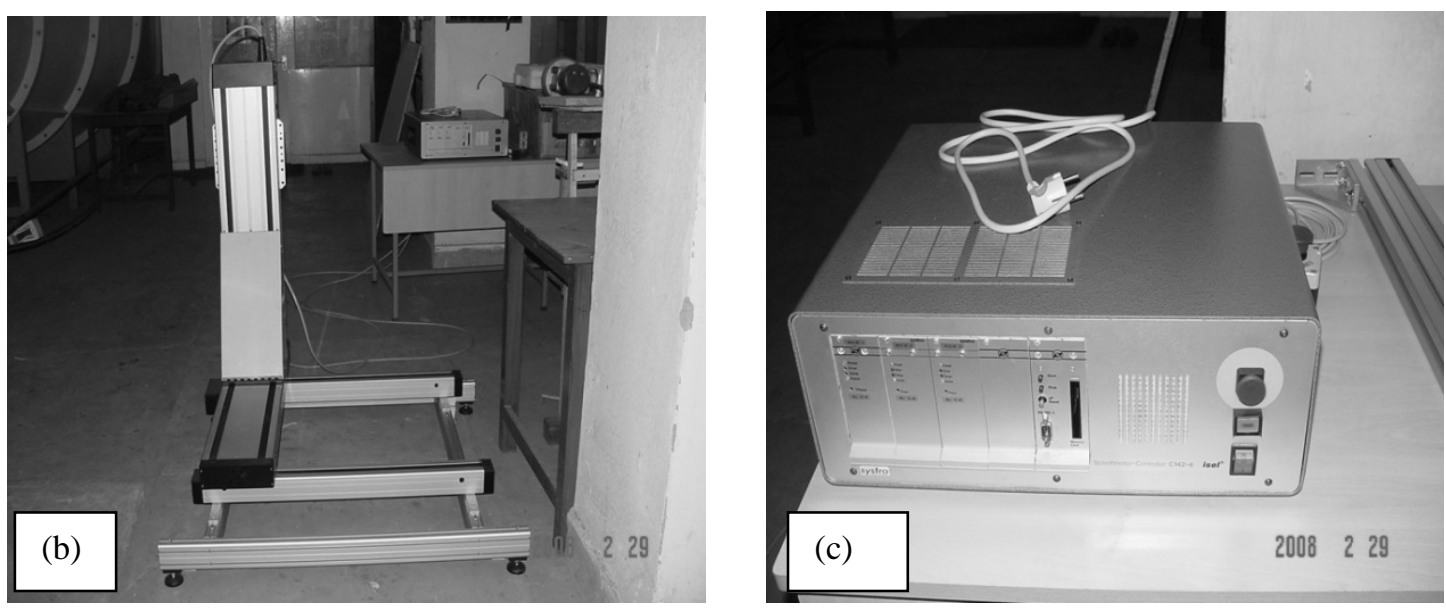

Figure 2 DANTEC CTA Probe with the Traversing system and the controller

\subsection{Flow visualization}

Flow visualisation of the impinging jet is done using a smoke generator and high speed cameras. Smoke generator is connected to the inlet of the blower. Flow patterns at different jet velocities and plate locations are photographed. Figures 4-7 shows the photographs of the flow visualisation taken at different time scale. A ring of circulation is clearly seen in the impinging jet prior to impact with the surface(Figure 4(c)). Formation of the primary and the secondary vortices are clearly seen from these photographs. High velocity region is visible near the impinging plate. Flow patterns seen in figures 4-7 of the flow visualisation in the physical simulator are very similar to photographs of a full scale downburst as shown in figure 10.
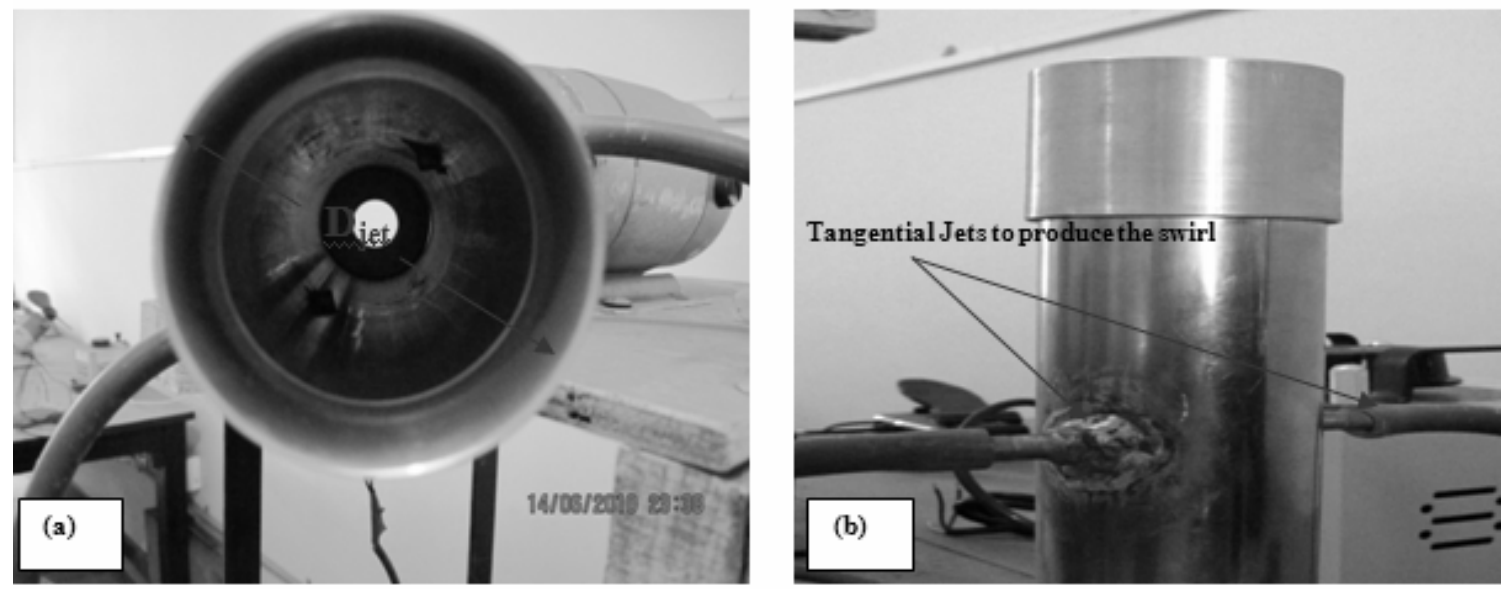

Figure 3 Impinging jet

\section{Results and Discussions}

Figure 4 shows flow patterns at different time level for the downburst simulator for jet velocity of $10 \mathrm{~m} / \mathrm{s}$ at $\mathrm{H} / \mathrm{D}_{\text {jet }}$ of 1.5 . Generation of the encircling ring vortex is seen in figure 4(c). Existence of a region having high velocity and vorticity is seen very close to the surface just like full scale downburst. Figure 4(f) shows the formation of the primary and secondary vortices due to initial Kelvin-Helmholtz instability. In the free jet region the initial Kelvin-Helmholtz instability generates the vortex rings because of stronger shear generated at the start-up as surrounding fluid is at rest. Similar results are also obtained for other jet velocities at different height/diameter ratio. The maximum radial velocity is seen in the region of $x=1.1-1.5 D_{j e t}$. Figure 5 shows the flow pattern of the downburst simulator for jet velocity of $15 \mathrm{~m} / \mathrm{s}$ at the same plate separation ratio 1.5. Figure 5 shows higher horizontal ring vortex than figure 4. Formation of the primary and secondary vortices is also seen in figure 5(d). Figure 6 shows the flow patterns for jet velocity of $20 \mathrm{~m} / \mathrm{s}$ at the same plate separation ratio of 1.5. Figure 7 shows very high magnitude primary and secondary vortices. So, it is seen that the magnitude of the primary and secondary vortices depend on the jet velocity(Reynolds 
number). Also it is seen from figures 4-6 that location of the primary vortex is different for differs slightly for different jet velocities. It is seen from figures 4-6 that for lower Reynolds number location of the primary vortex is closer to the plate. Hence we can conclude that radial velocity profiles depend on the Reynolds number of the flow. Radial velocity in the downburst flow is very high near the ground because in the impinging jet region the vortex rings touch down the surface and start moving radialLy triggering an unsteady separation of the newly formed boundary layer. The counter rotating primary and secondary vortices moving radially outward produce maximum radial velocity closer to the surface. Figure 7 shows the flow pattern for jet velocity 20 $\mathrm{m} / \mathrm{s}$ at plate separation ratio of 2.0. It is clear from figures 6-7 that the formation of the primary and secondary vortices differs for change of jet velocity(Reynolds number). It is seen from figures 6-7 that the locations of the primary and secondary vortices are closer to the plate for lower plate separation ratio. Figure 8(a) shows the experimental readings for the radial velocity profiles for the jet velocity $10 \mathrm{~m} / \mathrm{s}$ for plate separation ratios 1.0 and 1.5. From figure 8(a) it is seen that that magnitude and the location of the maximum radial velocity or the primary ring vortex depend on the Reynolds number of the flow. Location of the radial velocity is closer to the surface for lower $\mathrm{H} / \mathrm{D}_{\text {jet. }}$. Figure 8 (b) shows the experimental readings for the axial velocity profile for plate separation ratio of 1.0. Higher axial velocity can be seen in the region of primary vortex. Figure 9 shows the radial velocity profiles at a radial distance of $1.2 \mathrm{D}_{\text {jet }}$ for jet velocities of $15 \mathrm{~m} / \mathrm{s}$ and $20 \mathrm{~m} / \mathrm{s}$. It is clear from figure 9 that radial velocity profiles differ significantly for different Reynolds number. Maximum radial velocity is higher for higher Reynolds number. Figure 11 shows the measured and computed radial velocity from the code developed by Das et al.(2010) at a height of $0.1 D_{\text {jet }}$ from the plate for the configuration having $H / D_{\text {jet }}=R_{1}$ at Reynolds number $\operatorname{Re}_{1}$. The results are compared with the well-known correlation proposed by Holmes and Oliver (2000). Holmes and Oliver (2000) reported that the radial velocity profile using the empirical formula agrees well with the radar observation of the full scale phenomena by Hjelmfelt (1988) when radial length scale $(R)$ is $50 \%$ of $\mathrm{r}_{\max }$. Following the observation $R$ is taken as $50 \%$ of $\mathrm{r}_{\max }$ for the present comparison. Figure 12 shows fairly good agreement between the current CFD and experimental results and the results from the empirical formula. However, the experimental and computational data indicate that the radial velocity in the stagnation region is not truly linear as given by potential flow solution and used in Holmes and Oliver (2000). The subsequent decay follows the exponential law to a certain distance, nearly $1.5 \mathrm{r}_{\max }$ for the cases studied here. However, beyond this distance the velocity decay rate reduces considerably due to the influence of the secondary vortex generated just outside and below the main vortex ring. Thus, the secondary vortex helps to increase the radial extent of the downburst wind.
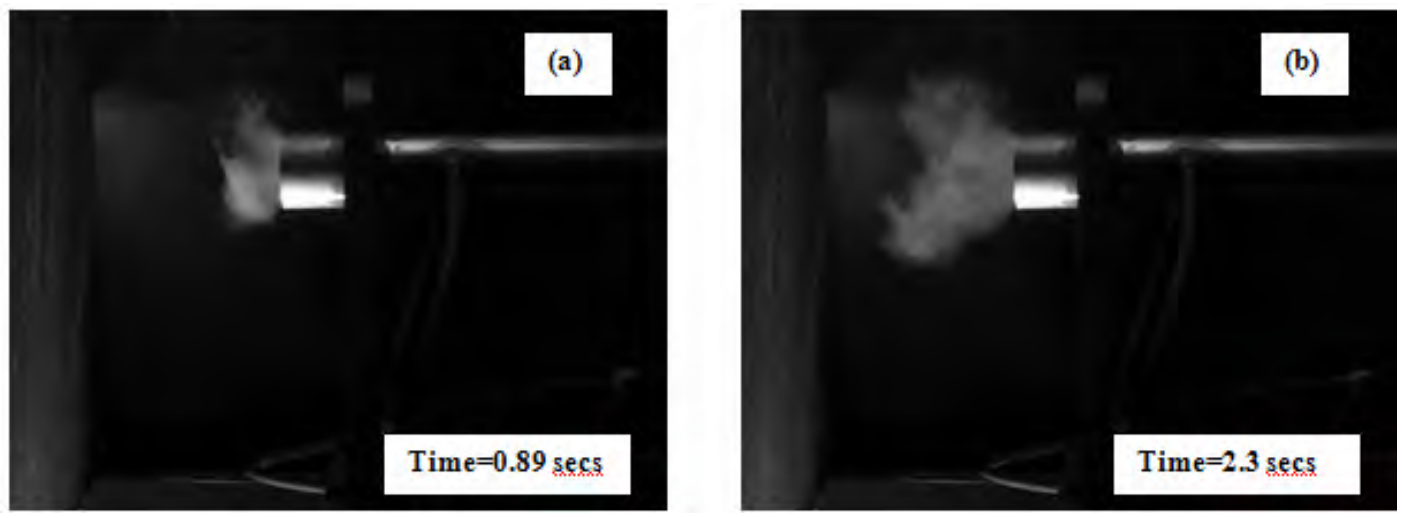

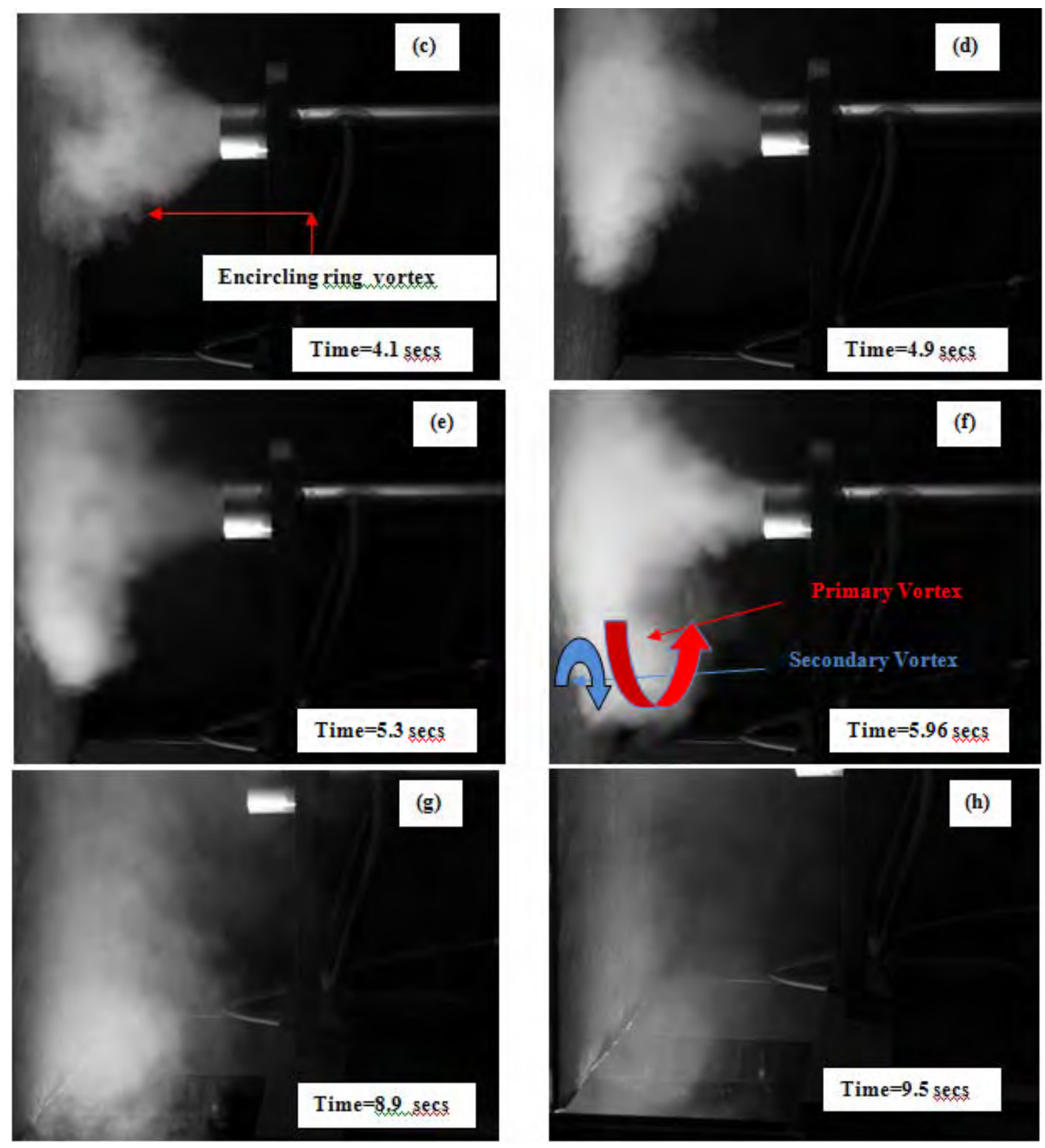

Figure 4 Flow visualization for $\mathrm{V}_{\mathrm{jet}}=10 \mathrm{~m} / \mathrm{s}$ at $\mathrm{H} / \mathrm{D}_{\mathrm{jet}}=1.5$ 

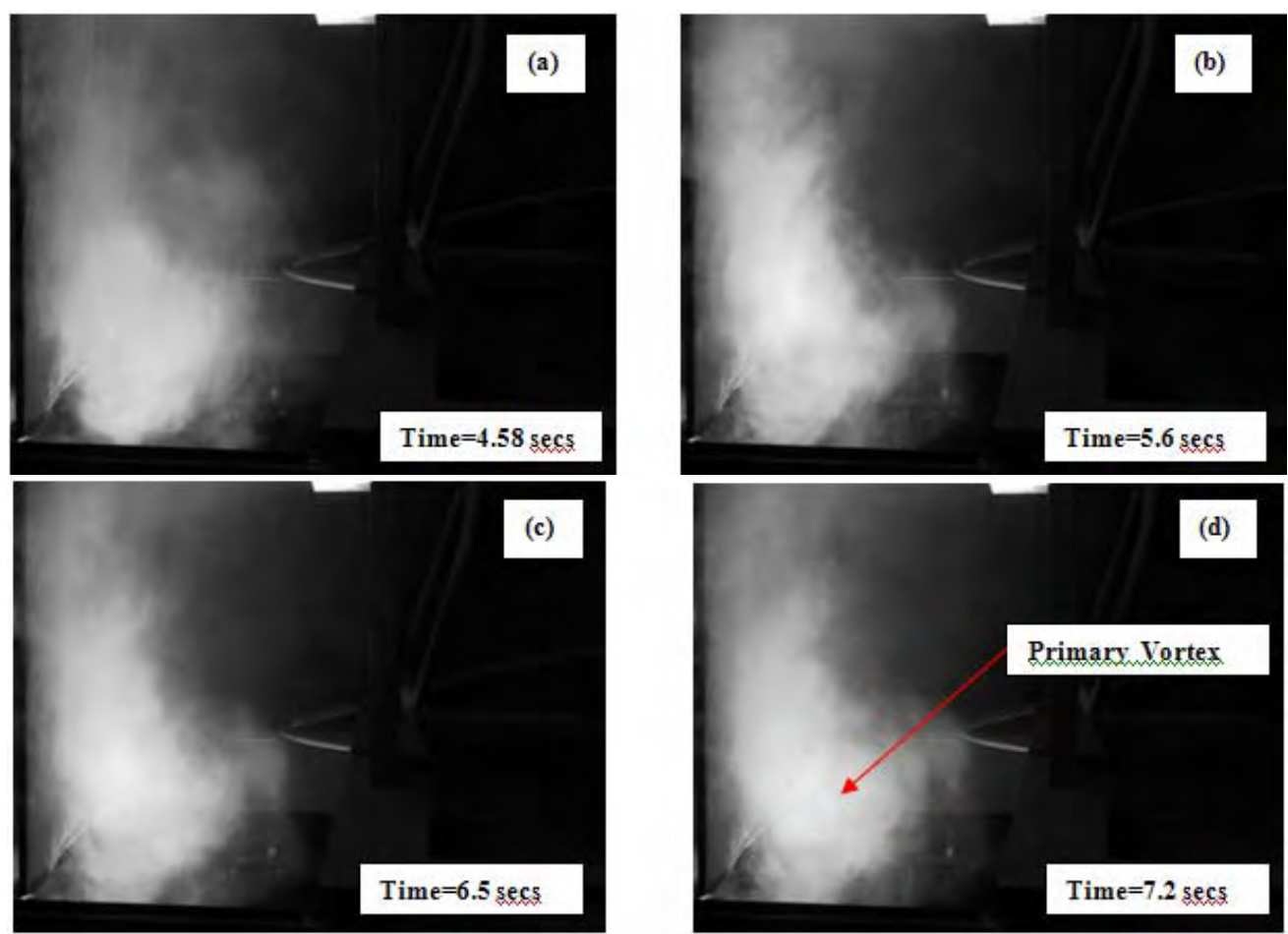

Figure 5 Flow visualization for $\mathrm{V}_{\text {jet }}=15 \mathrm{~m} / \mathrm{s}$ at $\mathrm{H} / \mathrm{D}_{\text {jet }}=1.5$
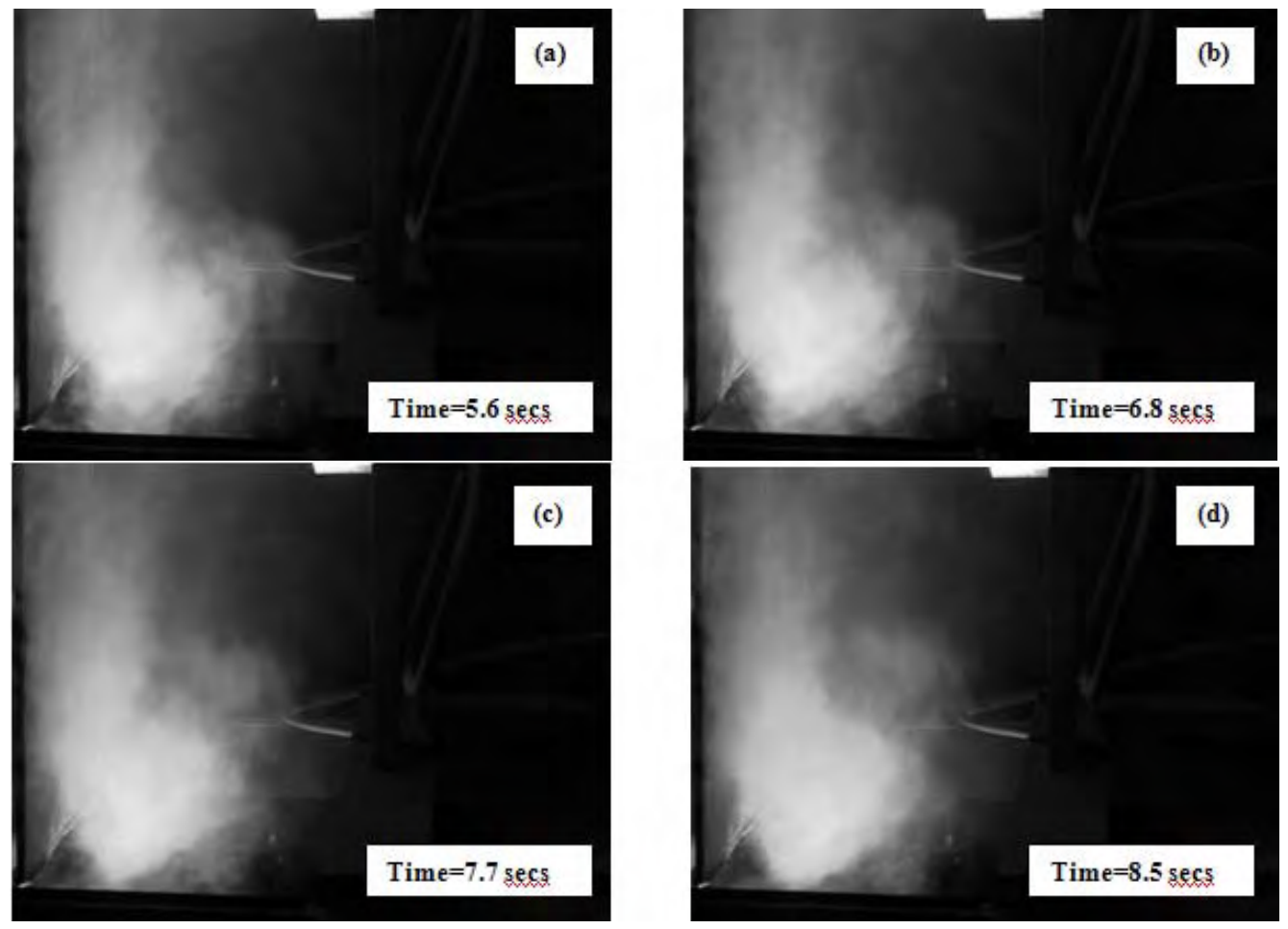

Figure 6 Flow visualization for $\mathrm{V}_{\text {jet }}=20 \mathrm{~m} / \mathrm{s}$ at $\mathrm{H} / \mathrm{D}_{\text {jet }}=1.5$ 

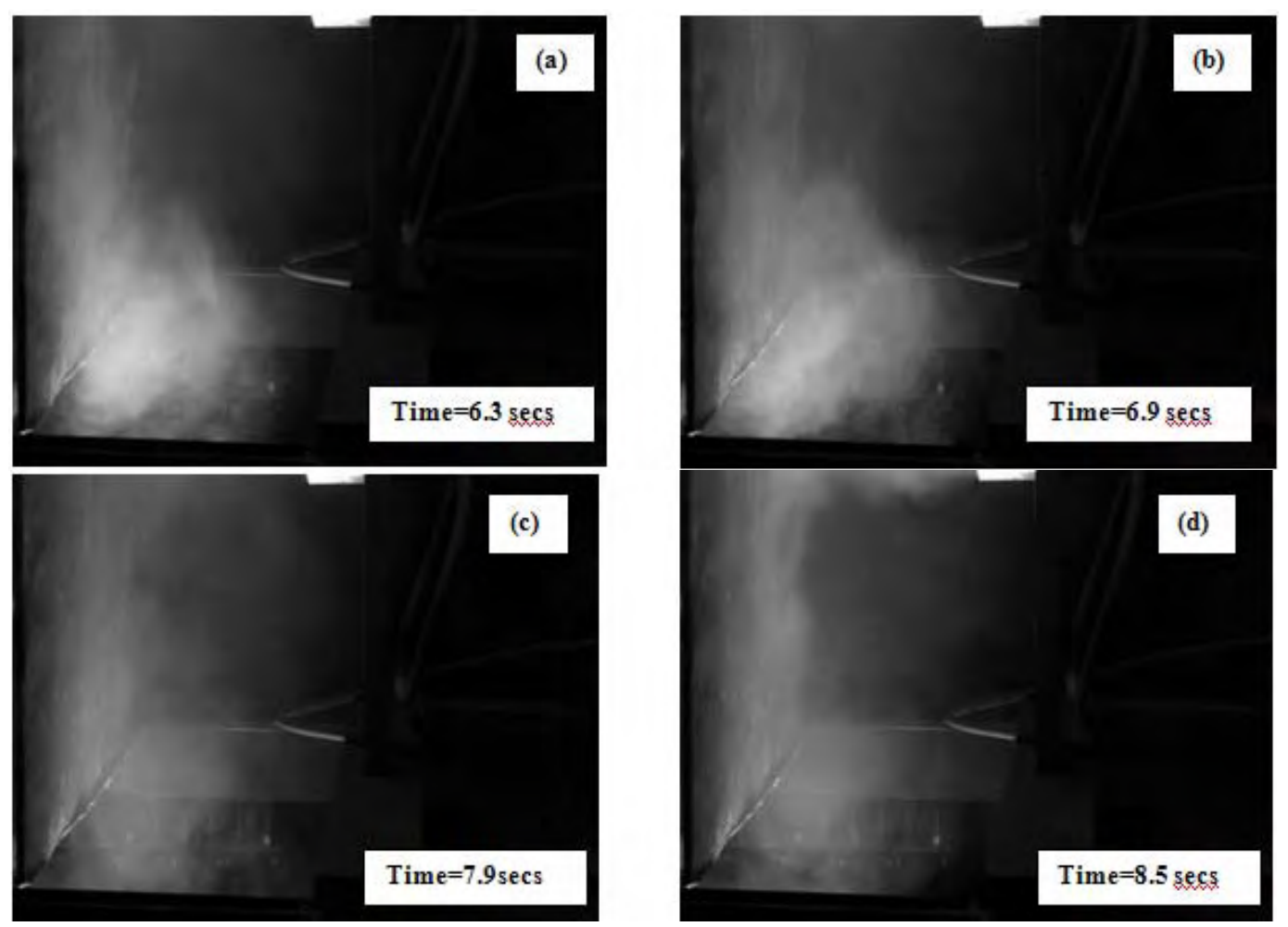

Figure 7 Flow visualization for $\mathrm{V}_{\text {jet }}=20 \mathrm{~m} / \mathrm{s}$ at $\mathrm{H} / \mathrm{D}_{\mathrm{jet}}=2.0$

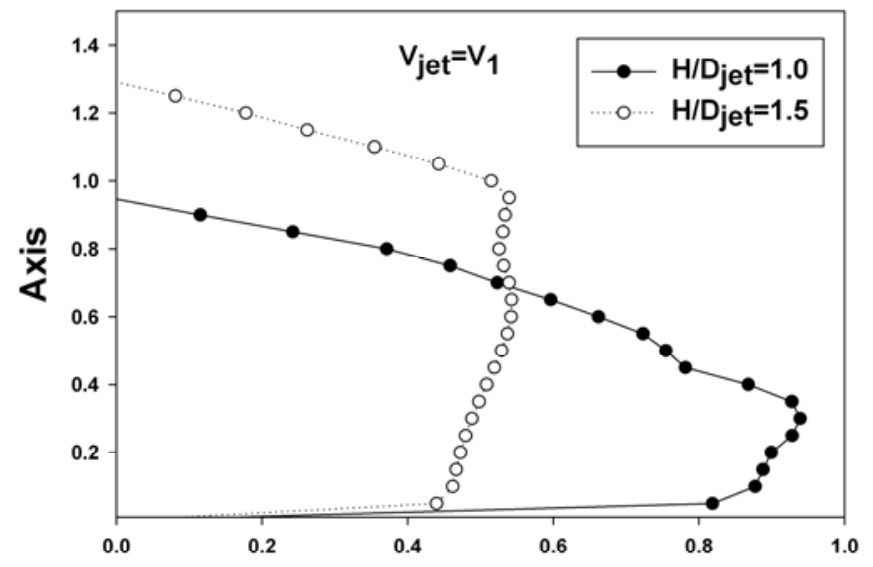

(a)

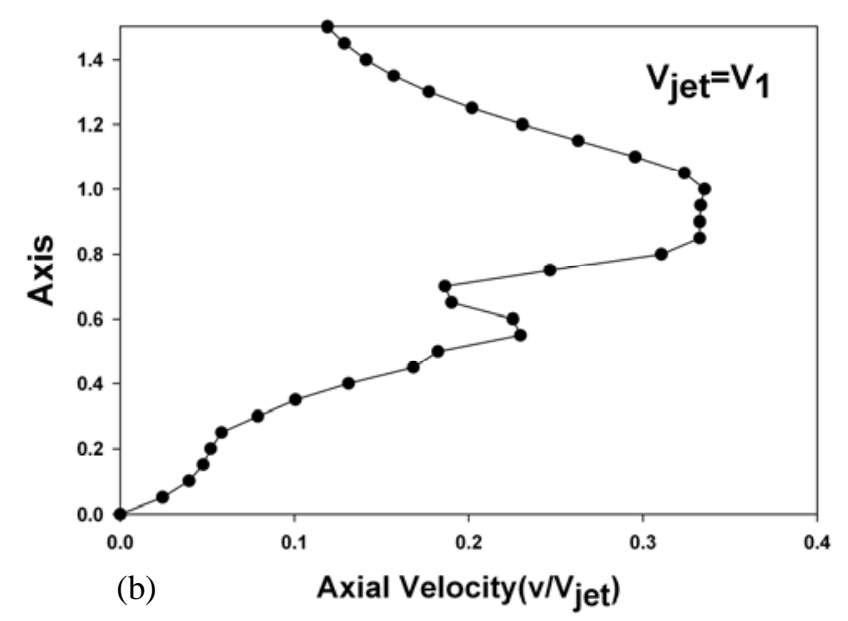

Figure 8 Experimental values of radial and axial velocity profiles for $\mathrm{V}_{\text {jet }}=\mathrm{V}_{1}$ at a radial distance of $1.2 \mathrm{D}_{\text {jet }}$ 

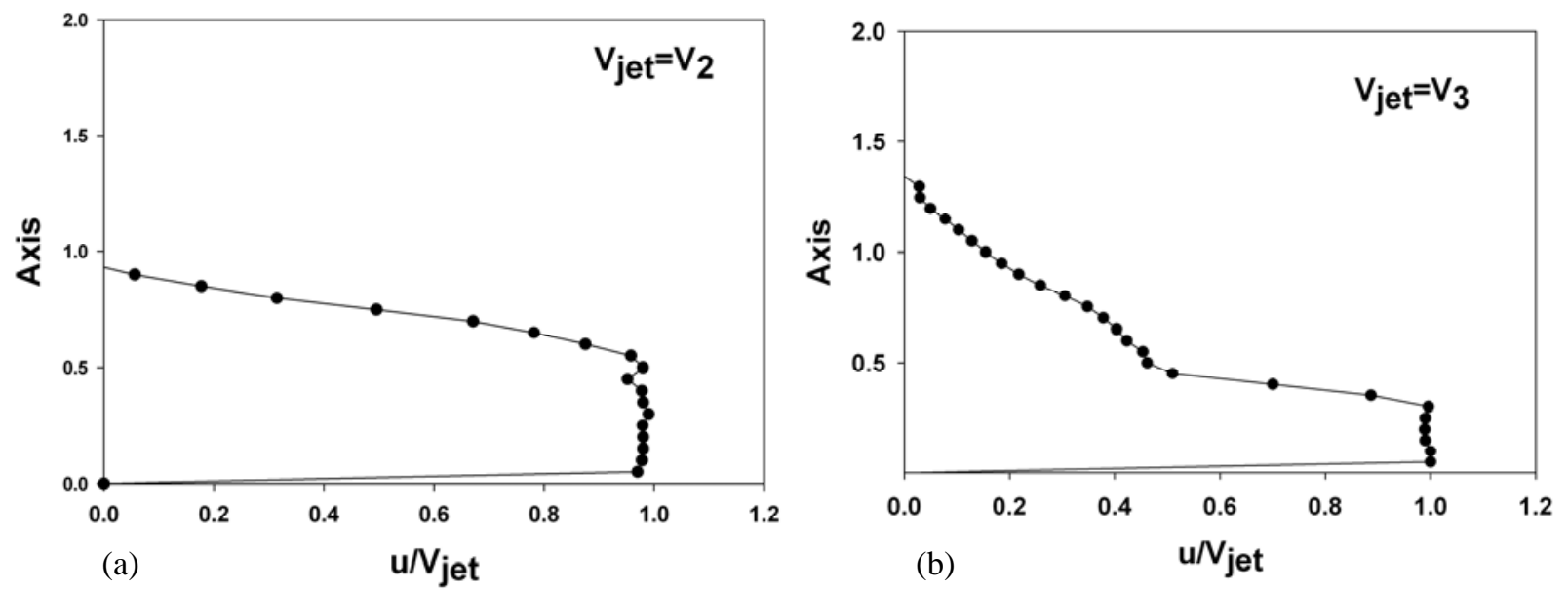

Figure 9 Radial velocity profiles at a radial distance of $1.2 \mathrm{D}_{\text {jet }}$ for different jet velocities
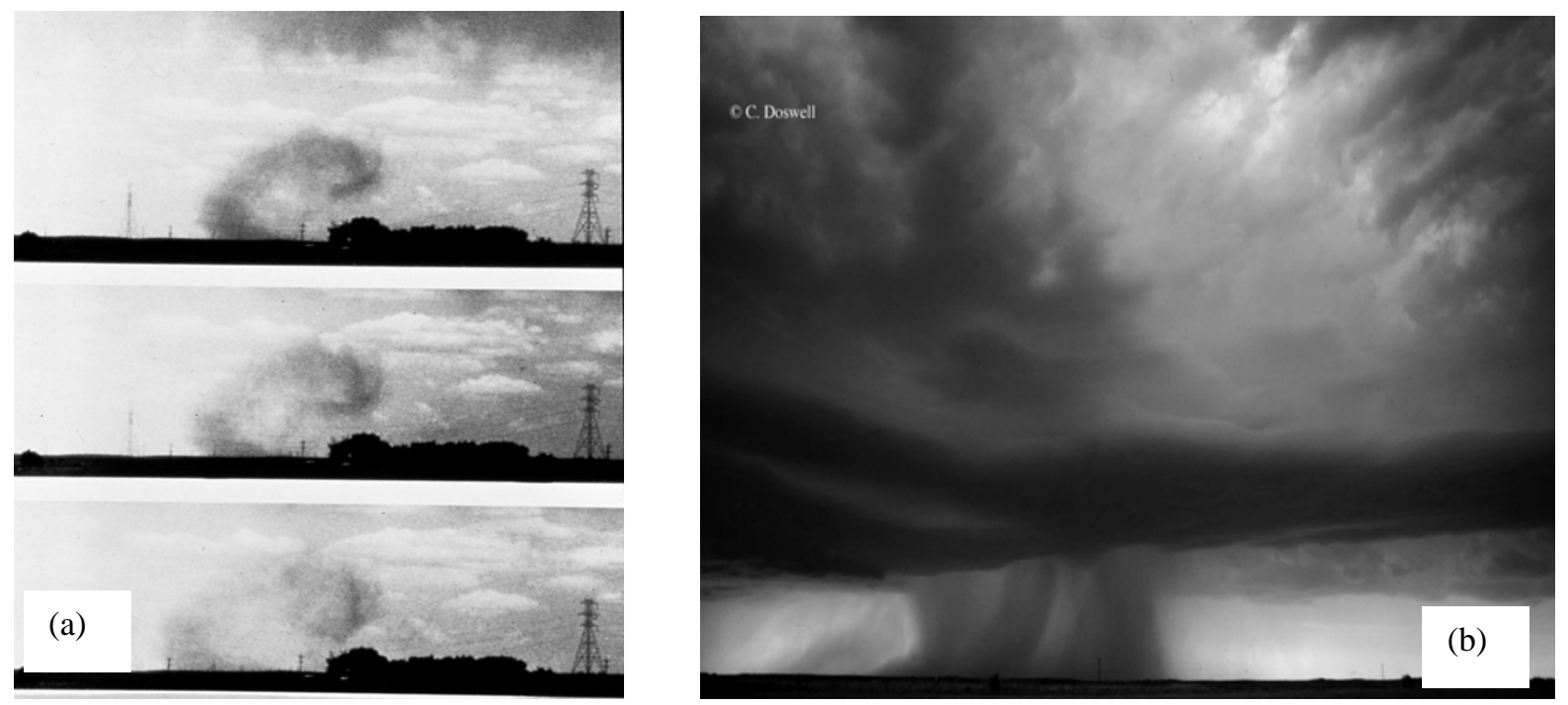

Figure 10 Picture of full scale downburst(Curtsey C. Doswell)

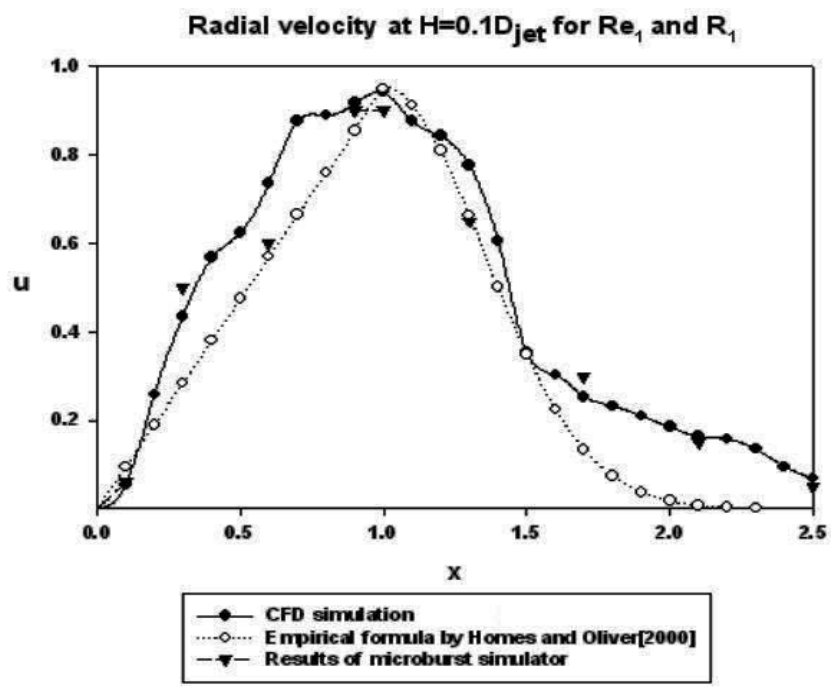

Figure 11 Comparison of results from the physical simulator with results of empirical relation of Homes and Oliver 


\section{Conclusion}

A physical simulator is fabricated for the laboratory simulation of the downburst based on the impinging jet model with facility to generate swirl in the jet. Radial velocity profiles for different jet velocities and impinging plate separation are investigated. Simulated downburst results are compared with the results from the empirical relation of Holmes and Oliver (2000) and good agreement is observed between these results. To study macro flow dynamics of the simulated downburst flow visualization is done using a smoke generator. The present study reveals the following facts regarding the downburst flow field,

(a) The maximum radial velocity of the microburst near the ground increases with the increase in Jet velocity and decrease in plate separation.

(b) The region of high radial velocity moves closer to the impinging plate for an increase in jet velocity and decrease in plate separation.

(c) Microburst flow field characteristics depend on jet velocity, plate separation and jet diameter.

(d) The expansion and touchdown of the encircling ring vortex reduces the radial velocity decay rate beyond $1.5 \mathrm{D}_{\text {jet }}$ and hence increases the radial extent of the downburst.

(e) The generation of the secondary vortex after the touchdown of the jet produces the maximum radial velocity before $\mathrm{x}=\mathrm{D}_{\text {jet }}$.

\section{Nomenclature}

$\mathrm{x} \quad$ Radial direction

y Axial direction

$\mathrm{V}_{\text {jet }} \quad$ Jet velocity

u Radial velocity

v Axial velocity

$\mathrm{D}_{\text {jet }} \quad$ Diameter of the jet

$\mathrm{H} \quad$ Distance of the jet from the impinging plate.

$\mathrm{H} / \mathrm{D}_{\text {jet }} \quad$ Plate separation ratio, Cloud height for the full scale downburst.

\section{References}

Alahyari, A., Longmire, E.K., 1995. Dynamics of experimentally simulated microbursts. AIAA J. 33 (11), 2128-2136.

Chay, M.T., Letchford, C.W., 2002. Pressure distribution on a cube in a simulated thunderstorm downburst—Part A: stationary downburst simulation. J. Wind Eng. Ind. Aerodyn. 90, 711-732.

Cooper, D., Jackson, D.C., Launder, B.E., Liao, G.X., 1993. Impinging jet studies for turbulence model

Assessment-I. Flow-field experiments. Int. J. Heat Mass Transfer 36 (10), 2675-2684.

Craft, T.J., Graham, L.J.W., Launder, B.E., 1993. Impinging jet studies for turbulence model Assessment-II: an ex amination of the performance of four turbulence models. Int. J. Heat Mass Transfer 36 (10), 2685-2697.

Das K.K, Ghosh A.K., Sinhamahapatra K.P.,2010, Investigation of the axisymmetric microburst flow field, Journal of Wind and Engg., Vol. 7 no. 1, Jan 2010, pp 1-15.

Didden, N., Ho, C.M., 1985. Unsteady separation in a boundary layer produced by an impinging jet. J. Fluid Mech. 160, $235-256$.

Fujita, T.T., 1981. Tornadoes and downbursts in the context of generalized planetary scales. J. Atmos. Sci. 38, 1511-1534.

Fletcher C.A.J 1987 Computational Techniques for the Fluid Dynamics(vol. 2) Springer-Verlag Publication

Hjelmfelt, M.R., 1988. Structure and life cycle of micoburst outflows observed in Colorado. J. Appl. Met. 27, 1988, 900-927

Holmes, J.D., Oliver, S.E., 2000. An empirical model of a downburst. Eng. Struct. 22, 1167-1172.

Kim, J., Hangan, H., 2007. Numerical simulation of impinging jets with application to downbursts. J. Wind Eng. Ind. Aerodyn. 95, 279-298.

Knowles, K., Myszko, M., 1998. Turbulence measurement in radial wall-jets. Exp. Thermal Fluid Sci. 17, 71-78.

Letchford, C.W., Chay, M.T., 2002. Pressure distributions on a cube in a simulated thunderstorm downburst, Part B: moving downburst observations. J. Wind Eng. Ind. Aerodyn. 90, 733-753.

Lundgren, T.S., Yao, J., Mansour, N.N., 1992. Microburst modeling and scaling. J. Fluid Mech. 239, 461-488.

Proctor, F.H., 1988. Numerical simulations of an isolated microburst. Part I: Dynamics and Structure. J. Atmos. Sci. 45, 31373160.

Sakamota, S, Murakami S., Mochida A., 1993. Numerical study on flow past 2D square cylinder by Large Eddy Simulation Comparison between 2D and 3D computations, J. Wind Eng. Ind. Aerodynamics 50 (1993) 61-68. 
Selvam, R.P., Holmes, J.D., 1992. Numerical simulation of thunderstorm downdrafts. J. Wind Eng. Ind. Aerodyn. Vol. 41-44, pp. $2817-2825$.

Sengupta, A., Sarskar, P. P., 2007. Experimental measurement and numerical simulation of an impinging jet with application to thunderstorm microburst winds. J. Wind Eng. Ind. Aerodyn.

Received October 2010

Accepted March 2011

Final acceptance in revised form May 2011 